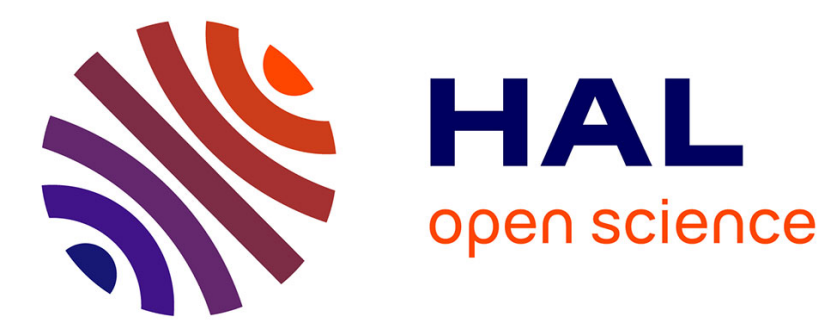

\title{
Educative Distributed Virtual Environments for Children
}

Dorin-Mircea Popovici, Jean-Pierre Gerval, Pierre Chevaillier, Tisseau Jacques, Luca-Dan Serbanati, Patrick Gueguen

\section{- To cite this version:}

Dorin-Mircea Popovici, Jean-Pierre Gerval, Pierre Chevaillier, Tisseau Jacques, Luca-Dan Serbanati, et al.. Educative Distributed Virtual Environments for Children. Journal of Distance Education Technologies, 2004, 2 (4), pp.18/40. hal-02999084

\section{HAL Id: hal-02999084 https://hal.science/hal-02999084}

Submitted on 10 Nov 2020

HAL is a multi-disciplinary open access archive for the deposit and dissemination of scientific research documents, whether they are published or not. The documents may come from teaching and research institutions in France or abroad, or from public or private research centers.
L'archive ouverte pluridisciplinaire HAL, est destinée au dépôt et à la diffusion de documents scientifiques de niveau recherche, publiés ou non, émanant des établissements d'enseignement et de recherche français ou étrangers, des laboratoires publics ou privés. 


\title{
Educative Distributed Virtual Environments for Children
}

\author{
Dorin-Mircea Popovici, ENIB/CERV, France \\ and OVIDIUS University of Constanta, Romania \\ Jean-Pierre Gerval, ISEN, France \\ Pierre Chevaillier, ENIB/CERV, France \\ Jacques Tisseau, ENIB/CERV, France \\ Luca-Dan Serbanati, OVIDIUS University of Constanta, Romania \\ Patrick Gueguen, Ecole Kroas Saliou, France
}

\begin{abstract}
This paper presents a distributed virtual reality environment for children called EVEEnvironnements Virtuels pour Enfants. The virtual environment architecture is reactive agents based. The FCM-like dynamic action planning mechanism assures agent's adaptability to its environment changes. This virtual environment supports cooperation among members of a dispersed team engaged in a concurrent context. By the means of their avatars, special cases of agents, users are allowed to interact and to give decisions using cooperative mechanisms. A user-friendly interface enables teachers to create their own stories that fit with children's pedagogical requirements and generate new virtual environments according to the teacher's specifications. The implementation is based on DeepMatrix as environment server, VRML and Java as languages and Cortona VRML plug-in from ParallelGraphics. It is actually running on the Internet: http://eve.enib.fr
\end{abstract}

Keywords: autonomous reactive agents, distributed virtual reality, fuzzy cognitive map, virtual environment

\section{INTRODUCTION}

Learning to read is a difficult but important task for a child. It takes time and supposes a constant effort from its part. Like many other school activities, reading involves child's capacities as attention and memory, knowledge (of letters) and knowhow as searching and discovering the word's sense in a given context. Small capacity in attention and incapacity to reinvest in a new task of already known notions are just some examples of obstacles in a child's learning process. More, a task failure can generate a fear from the child's part concerning particular notions, or notions related with the unaccomplished task.

At the beginning of primary school, some children have not achieved all the necessary acquisitions to basis tasks. For example, some have difficulties to place in order a set of labels in order to construct simple sentences. And this may have multiple causes; the child has not well memorized the oral sentence, is not able to realise a correspondence between the oral sen- 
tence and the written one, or, is not able to coordinate the spatial distribution from left to right with temporal succession of already pronounced words. In order to avoid the initial difficulties to accumulate and to produce a child's discouragement, part of the activity in primary schools is organised around small groups of children, rather than individual work. More, when we talk about learning at childhood age we are usually using a story metaphor, because narrative lends itself to active exploration of a domain through challenging and enjoyable problem-solving activities, which is essential for learning.

On the other hand, the informal context in which the child learns reading has well evolved in the past three decades. New technologies such as Internet, multimedia, and virtual reality are now parts of our children's everyday life. For this reason, it is not surprising that educators grow their interest in distance learning and distributed education, and try to use these types of media in their lessons.

Our paper presents a distributed nonimmersive virtual environment (VE), called EVE: Environnements Virtuels pour Enfants (Virtual Environments for Children), which helps primary school children learn to read. Based on a story reconstruction, it offers children a pleasant approach to learning by means of two games that implement emulation and cooperation.

In the following, after a brief stateof-the-art of educational computer aided environments for children, we present the EVE project from a pedagogical perspective. Next, we will make some considerations from an architectural point of view and we give some insights concerning the implementation of our project. Finally, we will make some conclusions based on the experience obtained during the project and we end with perspectives of our work.

\section{BACKGROUND}

With ever-increasing computational processing power, the rapid growth of the World Wide Web, and the ongoing construction of a digital communications infrastructure, the creation of distributed (immersive), multi-participant VEs running on the Internet starts to prove its usefulness in child education.

These learning experiences may come in many forms. Educational quiz-like or puzzle-like kids' games for ABC activities such as reading, reading comprehension, math, writing, and so forth, and digital libraries, such as QueryKids (Druin et al., 2003), are some examples of multimediasupported learning environments. The creativity, encouragement, and great motivation supported by interactivity in a social context permits passive children to become active. More, these experiences realise cultural enrichment of a child's knowledge (Pantelidis, 1995). Dedicated Web sites, like "J@rdin des jeunes branchés" (J@ardin, 2004), provide online learning activities that are combined with games and information for elementary school projects as a help to navigate on the main Web sites.

Much of the appeal for applying VR in education is derived from the observations of educational theorists (Bruner, 1986; Piaget, 1929) who have stressed the value of actualizing learning through making it more real for students. Learning is facilitated through the construction of concepts built from the intuitions that arise out of their direct experience of the environment. The opportunities for young users to visit places and interact with events that distance, time, or safety concerns would normally prohibit, the greater understanding of concepts through the creation of multi-modal metaphors or representations and the ability to 
scale and manipulate these representations (Winn, 1993; Youngblut, 1998) are the major benefits of applying VR in educational environments.

In the early 90s, narrative-based systems such as Oz (Bates, 1992; Mateas, 1997) or Virtual Theatre (Hayes-Roth \& vanGent, 1997) have been mainly developed for the purpose of entertainment. They propose story contents asking the users, particularly children, to accomplish some tasks.

CityGame (Volbracht, Domik, BackeNeuwaldand \& Rinkens, 1998) studies children's' ability to find physically and mentally one's way in the 2D and 3D environments. Round Earth (Johnson, Moher, Ohlssoon \& Gillingham, 1999) and QuickWorlds projects (Johnson, Moher, Leigh \& Lin, 2000) look at the issues involved in the use of projection based VR systems with children, specifically in the well known ImmersaDesk (Johnson et al., 2002) or CAVE VR theatres. The effectiveness of multiple representations in enhancing education in elementary school is investigated.

NICE (Roussos et al., 1999) implements a persistent virtual garden in which children may collaboratively plant and harvest fruits and vegetables, cull weeds, and position light and water sources to differentially affect the growth rate of plants. Their interactions constitute the story of the garden.

KidsRoom (Bobick et al., 2000) is another interactive narrative for children. Using images, lighting, sound, and computer vision action recognition technology, a child's room was transformed into a storybased world for play. Objects in the room became characters in an adventure, and the room itself actively participated in the story, guiding and reacting to the children's choices and actions. Through voice, sound, and image the KidsRoom entertained and provoked the mind of the child.

Systems as Storykit (Montemayor et al., 2000), Teatrix (Prada, Machado \& Paiva, 2000), Puppet (Marshall, Rogers \& Scaife, 2002) or GhostWriter (Robertson, 2002) go further and evaluate the use of $\mathrm{VE}$ and computer mediated communication technology in the domain of teaching children story writing skills. Based on improvisation as a social and perceptual experience, the children are expected to interact with each other, playing the roles of different characters, as in GhostWriter. In Puppet, children are allowed to play multiple roles in an interactive narrative: audience, actor, scriptwriter, and editor. They are able to both "dive in," taking on the role of a character in the drama, and to "step out," reasoning about a character's emotional states and goals, as well as reflecting upon their own character dialogue recorded while playing with the system as scriptwriter. While in Storykit children create physical immersive story environments using low-tech construction materials such as cardboard, glue, paper and ink pens, as well as high-tech smart objects, in Teatrix they are doing the same using a set of predefined scenes and a set of predefined characters. These characters may act on behalf of the children or autonomously. Each child will expect the story to evolve in reaction to her character's actions. So, their characters must act in a believable way, in order for the story creation environment to engage the children in an entertaining experience, which can meet the child's cognitive needs to interpret, understand and interact with the world in terms of stories.

As we can see, there are several VR environments that are applied specifically to elementary children learning. Based on a story metaphor, they encourage children 
to be co-constructors of narration, promoting in this way the deep, connection-building, meaning-making activities that define constructivist learning; or suppose the children's participation as actors.

In the environment we present in the following, we have adopted a story-based approach too. Unlike the mentioned projects, we ask the children to discover a proposed story, based on a set of images that are distributed in a virtual school. Each image is described by a sentence. At its turn, the sentence is constructed using five labels. In order to discover the story, the children have to reconstruct the sentences and after that to cooperate in order to propose a logical order of discovered images. This way, they learn reading by playing.

\section{EVE PROJECT: GENERAL DESCRIPTION}

The EVE project has started in 1998. Initially funded by the Fonds Francophone des Inforoutes (Francophone Information Highway Fund), EVE has involved nine partners (universities, primary schools and SMEs) from three countries: France, Morocco and Romania. Since 2000, EVE is used by elementary schools from partners' countries as a supplementary tool in teaching children to read. More, in November 2003 an experience between France and Japan was realised. Children from the primary school Kroas Saliou (Plouzané, France) cooperated with children from Ootsukadai elementary school (Yokosuka, Japan).

The target of the project is twofold:

1. To implement new cooperative working environments.

2. To initiate new products development such as pedagogical software for primary school children.
On a pedagogical point of view, the main goal is teamwork. Children from different classrooms and countries are involved in cooperative work. They have to achieve a common task together, hoping that this will encourage curiosity and respect in a multicultural framework, at a children's level, and not only.

On a technical point of view, the EVE project implements distributed virtual reality technologies. Software had been developed using Virtual Reality Modelling Language (VRML) and Java languages.

From both perspectives, we can define our project as a NICE-like one, being narrative, constructionist and collaborative. Even if it does not consume a lot of resources, like KidsRoom, it can offer to its young users great satisfaction because of their capability to transfer the virtual experiences in the real world and vice-versa.

Actual use of virtual environment is put into the context where social patterns outside the desktop are considered. In our case the social dynamics and physical presence even meant that the kids achieved an efficient cooperation in the classroom when they come to learn how to use the software and interact.

\section{Pedagogical Aspects}

The primary pedagogical goal is to instil in children the capacity to read and understand a text. If for the reading and phonemes association from six to 18 months are necessary, for text understanding the child needs a much longer period of time. The latter understanding may become more profound and subtle.

Very often children become able to read without knowing what they are reading. And this represents a problem in all learning processes. How can we resolve a 
problem in math if we are not able to read and understand its content?

If the children are enthusiasts at the beginning, this state of spirit is calmed down with the time passing because of the long time interval necessary to learn, by the difficulties met and by the mixture between them. It is strongly necessary that teachers adopt a proper strategy in order to diversify and refresh children's interest. Despite the diversity of existed and used educational tools such as books, libraries, movies, theatres, educational games, and so forth, the learning process seems to be to repetitive. And this takes place in the child's life, full of other activities, such as sports, playing an instrument, TV, video games, holidays...

The child need of diversity does not stop at the school's doors, and our day schools are plugged into this evolution.

\section{Two Games}

The EVE application has been developed in order to help primary school children to learn reading. It offers children a pleasant approach to learning by means of two games that implement emulation and cooperation. This way, EVE is involved into the VR-based education current, being a source of educational diversity and richness.

\section{Figure 1: The Labels Game}

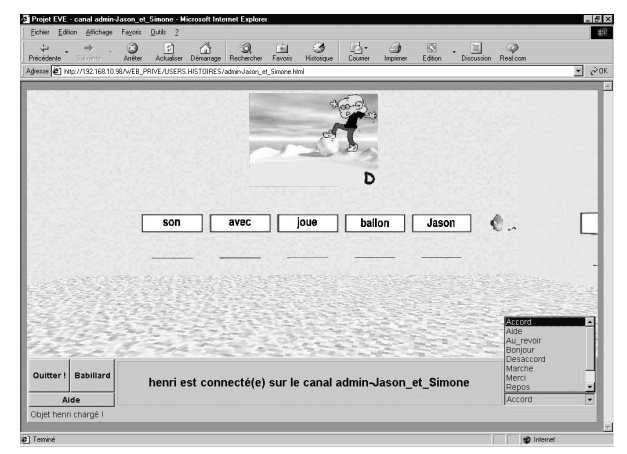

\section{The Labels' Game}

This first game is a self-training step according to a global learning method of reading, based on the label's game. The target of this first game is to build sentences. A picture illustrates each sentence, in order to suggest to the children the message of the sentence (Figure 1). The child must discover the sentence using a disordered set of words and moving words to the right places. In case of doubt, the child can hear the sentence by clicking on the appropriate icon. When the sentence is correct the child wins the picture. The child must win three pictures before being able to join the second game. Three children are working concurrently in three different virtual rooms, as orange, green and blue ones (Figure 2). The order in which the children finish their rooms is the same order they will have the right to express themselves in the next step. More, the first one will be allowed to start the second game. And this motivates them more.

\section{Let's Find Out the Story}

Several studies suggest that cooperation between children in virtual environments has a positive effect on learning (Johnson et al., 1999). It is not very unusual that performances that were expected without results in a child-adult context may

\section{Figure 2: The Virtual Environment}

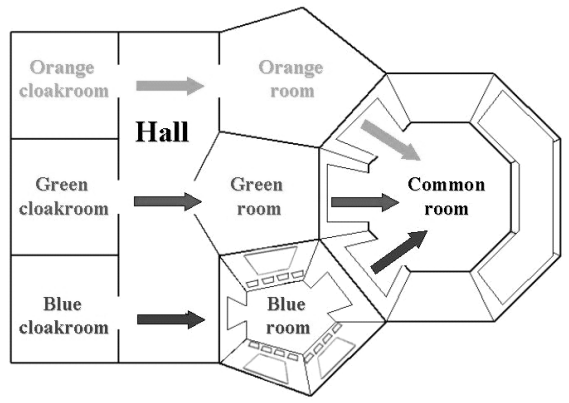

Copyright (C 2004, Idea Group Inc. Copying or distributing in print or electronic forms without written permission of Idea Group Inc. is prohibited. 
Figure 3: The Second Game -

Voting Mechanism

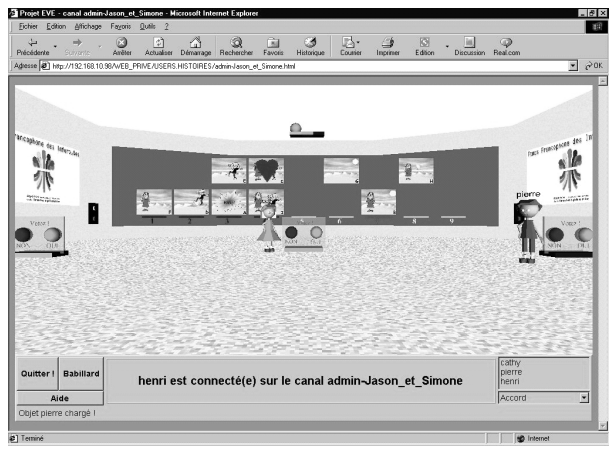

appear in a children's cooperative context. This is why collaborative learning is one of the most important requirements of our technology. Within a group of children, each child contributes with its knowledge in the group's knowledge, more or less easily acceptable by the others participants in its group.

In the framework of the second game, the spirit of the concurrence adds to the team spirit because of the cooperative context we propose to children. This time the children will meet each other in the common virtual room (Figure 2). Each child owns a personal avatar. The team of three children must now build a story. For this, they have to place the previously won pictures in a logical order. According to the order they have finished their rooms, they are allowed to move and propose positions for images. By viewing the moving of the selected images by the other children, the children are sure that they cooperate and they all have the same goal; even if they are in a neighbouring room, school or in a foreign country.

A special mechanism is needed in order to avoid conflicts: for example if two children want to move the same picture to two different positions. We have chosen to implement a voting mechanism (Figure 3):
Figure 4: Avatars' Behaviour

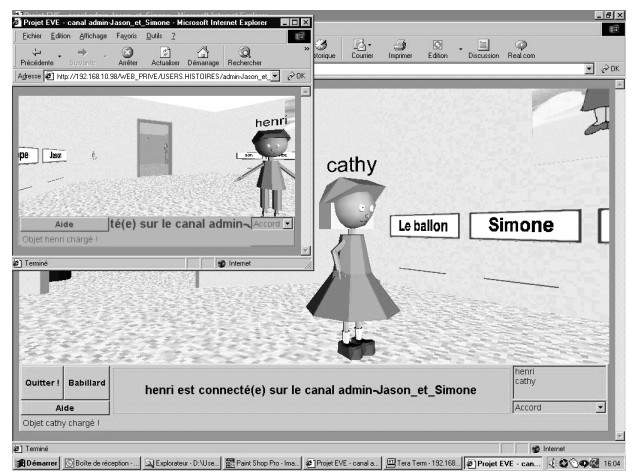

- A child moves a picture to a chosen position and requests a vote from the others.

- The two other children tell him if they agree or not. They are voting (green stands for yes, red for no).

- According to the vote result, the chosen position for the picture will be accepted or not.

When the team has finished building the story two cases may appear:

1. Pictures order is wrong. Badly placed pictures are removed and the team must start again to build the story.

2. Pictures order is right. An agent comes in the virtual common room and tells the story to the children.

This way, the success or the failure of the second game becomes a team result.

\section{Interactivity}

EVE supposes the ability of a child to find physically and mentally one's way in the 3D space. Spatial orientation is an important ability for understanding, interpreting and developing the world in which the children live. Children are given tasks of 
navigation and orientation inside the environment through intersecting paths in order to make them interact. The experienced children with three-dimensional computer games have certainly influenced the behaviour of the subjects.

One important result was that children often focused on the navigation tools (mouse and direction buttons) rather than on their tasks. More, they love to play "hideand-seek" and are eager to see what children in other rooms are doing. The application lets them to perform this task, because emulation concept is the basis of the first game. According to this fact, two mechanisms have been implemented:

- a scoreboard, which indicates the number of pictures that each child has won,

- a teleporting device, which enables the child to view what happens in other rooms.

A first idea when developing the EVE project was to implement a chat that enables children to discuss and explain their choices, especially during the second game. Previous experiments demonstrate that this choice was not suitable for the inexperienced in reading and writing learners. Actually, teachers are the main users of the chat!

By the mean of an avatar's behaviours (Figure 4) we increase the realism of the virtual representations of children playing in the virtual world and induce a better communication between children. We have chosen to implement behaviours for avatar actions as Walk, Standby, and used in children communication as "Hello!", "Help me!", "Thank you!", "I agree," "I disagree," Laughing, Smiling, and "Good Bye!" These previous lists are not exhaustive and should be easily completed according to current experiments with teachers and children. The child may select behaviour, which will be broadcasted (gesture and sound) to other children through it's the child's personal avatar.

In order to increase the interactivity level, some of the subjects suggested populating EVE with more elements to interact with.

\section{Agents in EVE}

In the very first stages of our project we have limited the number of children able to participate in a working session to three, according to the number of partner countries involved into the project. This was a strong constraint for children. Even the teacher proposed a certain story to play, children love some stories more than others, and usually there are more than three children that want to play the same story in the same time. So, in order to give to children the freedom to use the environment we have chosen two solutions.

\section{The Local Version}

In the local version of EVE, the task is basically the same except that there are no other children to participate in the voting mechanism. This means that the child has to complete all the three rooms in order to have access in the common, but empty, second room. Walking through the environment enables children to get use of the VRML plug-in functionalities before starting a distributed working session with other children. This way, a story may be used by as many children as possible.

\section{Using Virtual Agents}

Cooperation is an important concept of our technology. Suppose that the children are currently playing in EVE and for some unexpected cause a child is determined to quit the game. In such situations, the game cannot continue because normally 
Figure 5: Cultural Exchange through Language

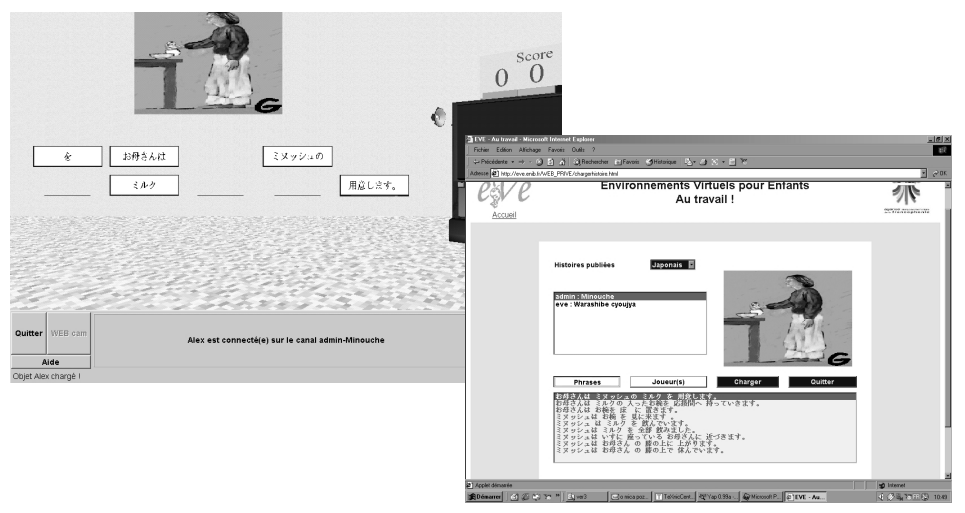

there is nobody to take the released place. In order to avoid this type of blocking, we have introduced a new user type, the virtual agent, which coexists within the environment and acts in the absence of a child. Doing so, the child can quit the game without affecting its flow, its role being switched to the corresponding virtual agent.

Based on virtual agents the number of users restriction falls. In other words, there are still only three children able to move labels and to vote in the same story, but we do not block the participation of other children as before. This way, the child is able to start alone a distributed working session, based on the cooperation with the existing agents. When there is another child who wants to participate, it is accepted as the second child, and the two children will continue the game. Finally, when the third child arrives in the environment it is accepted and the team is completed. From now on, all the other children will be accepted just as simple participants; they cannot express themselves by voting but they are allowed to cooperate (i.e., move labels) with others.

More, as in real life, the teacher and parents are represented in the environments, based on their special agents. They are the main users of the chat. While the parent's associated agent is not different from the child's ones from a behavioural point of view, the teacher's one is. As we will explain more in detail later, the teacher's agent is able to assist the child in its game. And he or she will do this by telling the sentence or even the whole story.

\section{Cultural Exchange}

Another dimension of EVE project is the cultural one. The proposed story-based metaphor facilitates the possibilities both for teaching familiar material in new ways, and presenting completely new material. So they are potentially of great value to education.

In order to facilitate and so to encourage the multi-cultural exchanges, we have extended EVE to support multi-language working sessions. This way, children can start learning foreign languages, or at least having a first contact with them, using the proposed stories. For the moment, in EVE we have stories in French, English, Romanian and even in Japanese (Figure 5).

\section{Children \& Teachers Together into an Interdisciplinary Team}

Children can play an important role in creating new technologies for children 
(Druin, 1999). Therefore, we have established a geographically distributed team of computer scientists, educational researchers, visual artists, primary school children and their teachers. During discussions among teams' members, children and teachers gave a strong feedback concerning stories' subjects, their graphic representation and audio support. More, the children were involved, at their demand, in audio and graphical support of stories' creation process. This way, we were able not only to understand the children's impact onto designing process, but we have also understood how the new technologies can impact children as users, and even as creators of these technologies.

In addition, the integration of primary school teachers as environmental and pedagogical designers has permitted us to realise a balance between technical and pedagogical aspects of our project.

\section{DESIGNING CONSIDERATIONS}

For the development of EVE as educational application, we have considered technological, domain specific, pedagogical and psychological aspects. First and foremost we have considered that in order to develop an application for real use in classrooms, we need an extendable system as a platform. And there is no single technology that fits all the application and educational needs. More, pedagogic and didactic skills were needed to adapt the application to the user requirements. Because the primary school children are the most part of our users, we have reduced at minimum the necessary skills to be trained or enhanced, like spatial abilities, but we have considered a number of psychological aspects. All these considerations have influenced content design, user interface's design and concepts for evaluations.

\section{The Environment}

The VE consists of a set of rooms that creates a simple maze (Figure 2). The environment wall's textures are based on real images from the Kroas Saliou primary school; wall paintings made by children, or on traditional French, Romanian or morocco images. Doing so, children have found the environment familiar, when even from architectural point of view it was not so. Visibility and accessibility aspects were taken into account also. As the three children have chosen one colour to log in EVE, orange, green or blue, we have use the same login colour in order to highlight the rooms through which they have to pass. More, we have matched the rooms' dimensions with the children's avatar ones.

Cooperation is basically supported by directly embodying the users in the VE using different avatars, and providing them with different inter-user communication facilities such as a set of predefined behaviours.

As constructivism underlines (Mantovani, 2001), learning takes place when learners can build conceptual models that are both consistent with what they already understand and with the new content. In order to ensure the successful adaptation of old knowledge to new experience, we have provided some flexible learning directions. The first one is the integration of different types of information and educational support other than $3 \mathrm{D}$ representation (such as audio annotations and images). More, we have defined specific tasks to the children through interaction. Participants are able to move their avatar around the rooms using the arrow keys and/or using the mouse. They are able to 
Figure 6: Story Data Model

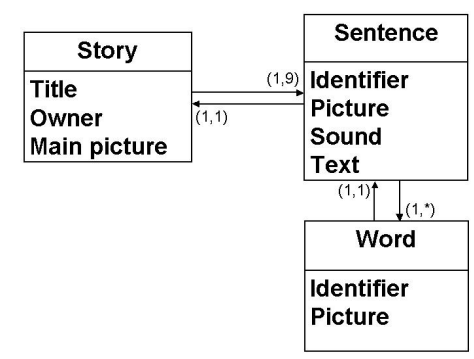

pick up objects (labels, images or speakers) in the VE by click-and-drag or just click them. Participants can communicate with each other using a chat channel.

\section{The Story}

A story is defined by an ordered set of nine sentences. As previously mentioned, a picture illustrates each sentence. A sound file is also linked to the sentence in order to enable children to hear the sentence. A sentence is defined by an ordered set of words. As it is presented, (Figure 1), a picture is associated to each word. The story data model is presented hereafter (Figure 6). According to this data model, which is really simple, we have chosen to implement this model using a simple directory structure.

\section{The Users' Interfaces}

Another challenge was to provide teachers with a user-friendly interface (Figure 7) that enables them to build their own stories. Using this tool, teachers can create stories that fit children pedagogical requirements all along the school year. Teachers can also involve children in designing new stories for their friends.

For each sentence, the teacher will choose: a gif file (the picture) and a wav file (the sound), and write the text of the sentence.

When this job ended, the teacher saves the story on the server. Before proceeding to data transfer, the application automatically:

- Generates a gif file for each word taking into account word length in order to use the best character font size;

- Transforms each wav file into an mp3 file in order to reduce the amount of data.

The teacher can clear the interface, and load or delete a story from the server previously created.

A new virtual world that implements the new story is generated when the teacher decides to publish its story. On the server side, symbolic links to the virtual world are created in the new story direc-

Figure 7: Story Interface - TOP (the left), BOTTOM (the right)
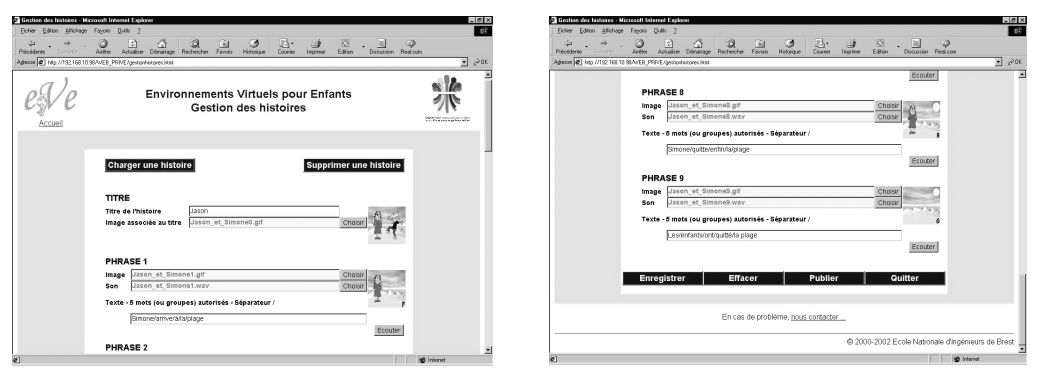

Copyright () 2004, Idea Group Inc. Copying or distributing in print or electronic forms without written permission of Idea Group Inc. is prohibited. 
Figure 8: Moderator's Interface (the left) and the Child's One (the right)

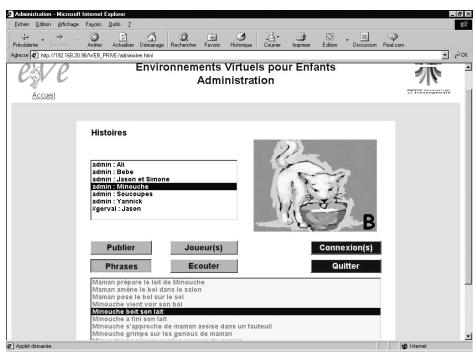

tory structure. At the same time, an e-mail is sent to a moderator.

A special interface (Figure 8, left) has been implemented that enables the moderator to view the story and to hear sentences. The moderator will read the new story and decide whether he or she accepts it or not according to its content.

The last interface (Figure 8, right) enables children or teachers to choose a virtual world. The list of all published stories is shown on the left upper corner of the page. The user:

- Chooses a story in the list;

- May click on the picture to view all pictures;

- Can press a button to read sentencessentences disappear when the mouse is released.

This interface enables the teacher to read the story before he or she starts working with the children.

\section{VIRTUAL \\ ENVIRONMENT'S ARCHITECTURE}

In EVE, we consider that the virtual environment is populated by virtual entities that correspond to objects populating the real world. In our vision, the "meaning" of each virtual entity is its associated object

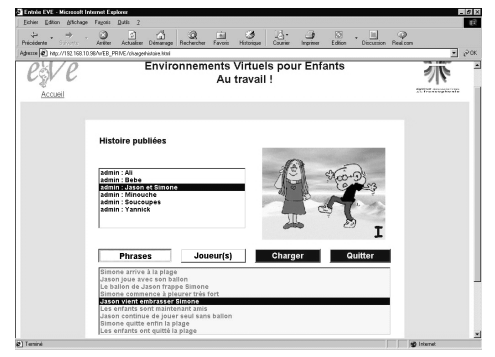

in the real world. As we know, in order to model an object in the real world some essential properties of the object should be used. They will make up the object's meaning from the modeler's point of view. In the virtual environment, for each property of the real object the modeler considers essential will be an attribute of the virtual entity with its informational shape, so the real object's property is for us the meaning of the attribute associated shape.

In the following, a virtual entity, briefly called entity, is the set of all informational shapes, as introduced in Popovici, Serbanati and Harrouet (2003), which complete its meaning (Figure 10). By using various criteria, we can structure the set of entities within the virtual environment. For example, the entities can be specialised in receptors and effectors. A receptor is a stimuli detector in an informational space, while an effector realises shape modifications by means of the entity's actions in the virtual environment. The entities may be grouped in order to produce complex aggregations, themselves entities. In the following section the agent as an aggregation of entities is presented.

\section{Agent's Architecture}

In our approach, a virtual agent is a complex entity in the virtual environment able to perceive, decide, and react based 
on its profile, internal structure, and tasks. Users' avatars are particular cases of virtual agents.

In order to realise the agent's setting in situation into the virtual environment we have followed the immersion, the interaction and the autonomy principles (Tisseau $\&$ Harrouet, 2003). For this, we were inspired by the human perception mechanisms (Hefco, 1997), as Herrero uses (Herrero \& Antonio, 2002). Our approach uses perception and emission fields of the entities, which are generalisations of medium, nimbus, aura, and awareness notions as they were introduced in Benford and Fahln (1993). The entities are supposed to populate a multi-dimensional informational space. This space is called virtual environment and it is viewed as the union of all entities' fields.

The interactions between agents are of cause-effect type: any change of the agent's state represents a possible cause and may be followed by a stimulus emission. In our approach, the stimulus contains information regarding the agent state (Popovici, Serbanati \& Gerval, 2003). All the agent's actions are followed by stimuli emissions.

Our virtual agents are autonomous in the measure of their adaptability to their dynamic environment. For this, they have to be able to perceive the environment changes, to decide and to react accordingly (Figure 9). A more detailed view of the agent's architecture is given in Figure 10.

During the virtual agent's life, its state is given by the values of its attributes that are the generators of its structure. The structure's variations are produced by the effectors and are perceived by means of the receptors, under the form of stimuli. These modifications may be triggered by the reception of an external stimulus such
Figure 9: Agent's Architecture

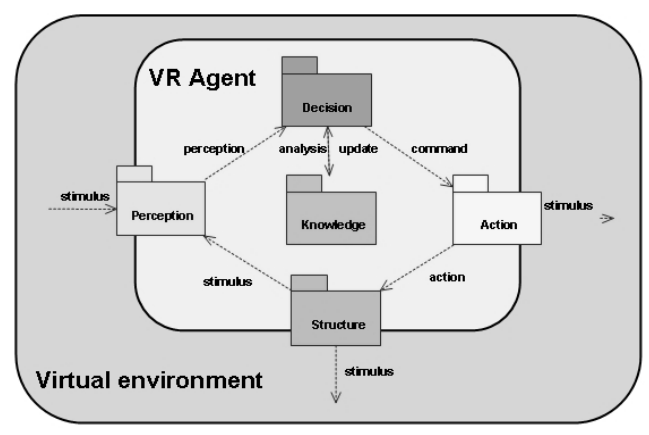

as a change in environment followed by emission of internal stimuli.

The receptors from perception module generate perceptions based on these stimuli. The resulting perceptions will be further used by the decision component, which is responsible for effectors' activation. In their turn, effectors, founded in the action module, may operate structural modifications followed by stimuli emissions, or may trigger themselves specific stimuli. This way, the agent's life cycle is completed.

Information concerning the agent's objectives and abilities, as well as its world model, is stored in its knowledge base. This is the only component which is modifiable not by the means of the agent's effectors but the decider one.

The problem that arises concerns the dynamical aspect of the agent's environment. In such an environment, the agent's decision based upon its actions may become obsolete between the moment the decision is made and the moment the action is applied. And this is due to the fact that in this time interval the agent's action selection mechanism does not update the activated action context. In order to eliminate this inconsistency we use a dynamical FCMlike action planning mechanism, based on agent's perception, situated at decision level. 
Figure 10: Inside View of the Agent's Architecture

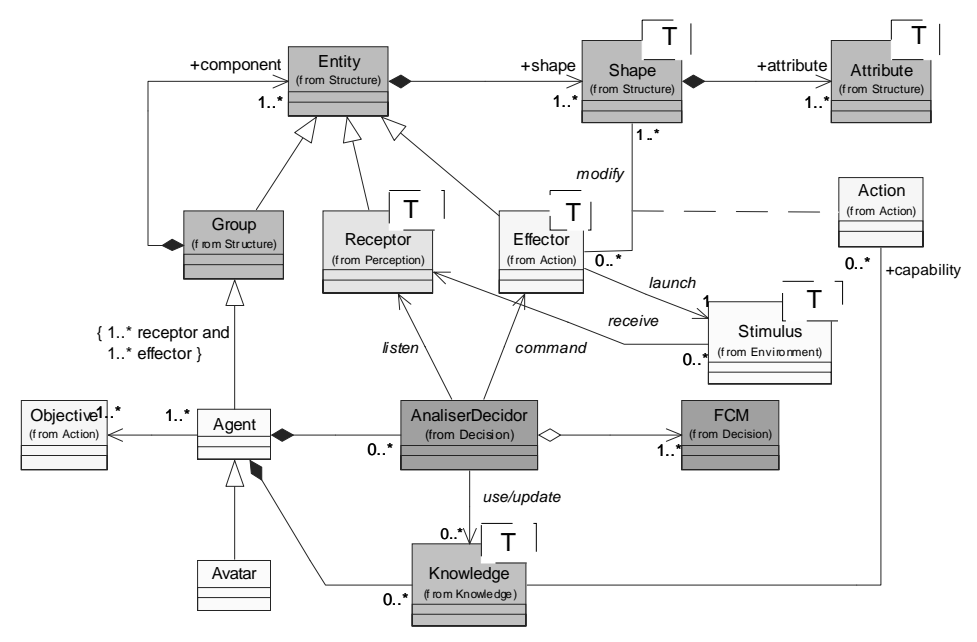

\section{Agent's Decider Module}

Using fuzzy cognitive maps (FCM), instead of the hierarchical task networks (HTN) (Cavazza, Charles \& Mead, 2002), we are able to ensure the agent's adaptability to environment changes. A FCM is an influence graph having as nodes elements of a set of concepts (Kosko, 1997). Concepts may be sensorial concepts (if they express perception values), internal concepts (for knowledge or decisional values), or driving concepts (for actions/objectives values) that the agent possesses (Parenthoen, Reignier \& Tisseau, 2001).

The environment perturbations detected through the agent's receptors will represent imposed values of sensorial concepts. This way, each time the FCM is computed, the agent's behavioural (re)activation is realised.

Let us consider the following FCMs, associated to the agent that plays the role of the tutor in EVE (Figure 11). Here, the sets of FCM concepts are "obstacle left," "obstacle right," "child far," "child close," "good answer" and "bad answer" as sensorial concepts, "motivation to approach" and "need to assist" as internal concepts, and "turn left," "turn right," "approach" and "assist" as driving concepts. "Advice," "valid sentence" and "valid story" are also driving concepts but, as we shall see in the next section, they are used in "assist" action plan. This means that they are not in-

Figure 11: Parts of the Tutor's FCM

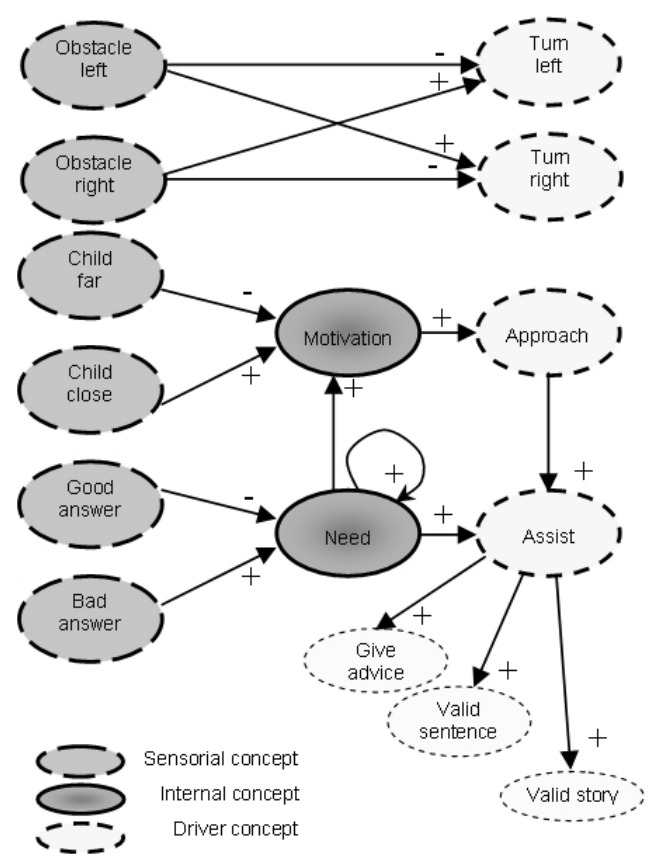


volved in the decisional process but the behavioural one.

In our model, not only the agent's objectives are viewed as fuzzy goals (ElNasr, Yen \& Ioerger, 2000), but also the action plans. This is because they all have corresponding driving concepts in the decider's fuzzy maps. An action with its corresponding driving concept greater than a specific value is called active; otherwise the action is called inactive.

An action's plan may include different "routes" the agent can follow in order to complete the action. Even if it is unique, the plan's execution can provide different solutions for the corresponding action, depending on the current context.

For example, in order to assist the child, the tutor can tell a sentence, or recall a grammar rule, or highlight the error. Another way to assist the child is to tell a sentence and highlight the error, and then to recall a grammar rule, depending on the child responses. We use three behavioural operators, "all," "first of' and "sequence" in an action plan expression. To briefly describe them, let us denote by wait the action with any arbitrary effect (i.e., any set of resulting stimuli) on the agent's state/ structure and which is by default accomplished, and by none the action that is never accomplished. We will denote by $A$ the set of an agent's actions, by $A *$ the set $A$ $\{$ wait $\}$, and by Time a linear temporal structure assumed to be discrete for the sake of simplicity.

We define the $A L L$ pattern by means of the binary operator " all" $\otimes: A^{2} \rightarrow A$ with the following semantic: we say that an action $A_{\text {res }}=A_{1} \otimes A_{2}$ is completed, and so the associated context is validated if $\exists t_{1}>t_{0} \in$ Time for which both $A_{1}$ and $A_{2}$ are completed in the moment $t_{1}$. Here $t_{0}$ denotes the moment of parallel activation of
Figure 12: ALL Pattern's FCM

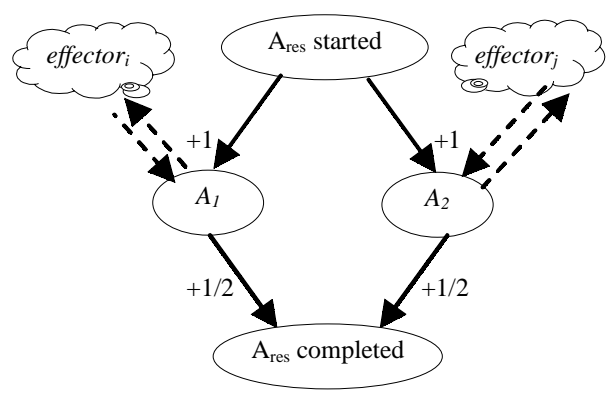

Figure 13: FOF Pattern's FCM

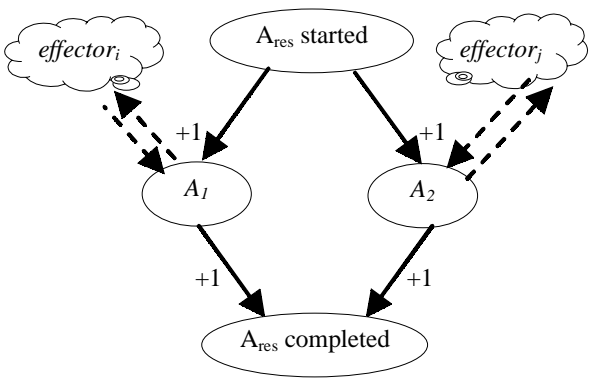

Figure 14: SEQ Pattern's FCM

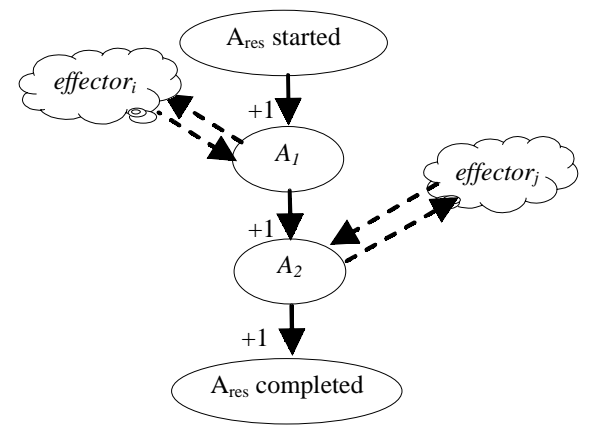

the actions. Its associated FCM is given in Figure 12.

Here, the set of FCM's concepts will correspond to the action's components, actions themselves. For each of the agent's effectors there will be one action that the effector controls. This means that an activated action will not activate its influenced actions (i.e., the actions activated by the current one in the FCM) until the corre- 
sponding effector completes its job. In Figure 12 , we have associated the effector to the action $A_{1}$ and the effector ${ }_{j}$ to the action $A_{2}$. With $A L L$ pattern we can express parallel cooperative actions (non-sequential).

Using the same FCM structure but with different influence values we obtain another binary operator, the "first of" $\oplus: A^{* 2} \rightarrow A$, which gives us the $F O F$ behavioural pattern. Its semantic is the following: we say that an action $A_{\text {res }}=A_{1} \oplus A_{2}$ is completed if $\exists t_{1}>t_{0} \in$ Time and $\exists j=1,2$ such that $A_{j}$ is completed at the moment $t_{l}$ and $\forall k=1,2$, and $A_{k}$ is not completed before, that is, at any moment $t, t_{0}<t<t_{1}$ (Figure 13). In this case, the context consists in the first accomplished action's effect. We use the FOF pattern when parallel concurrent actions (non-sequential) are needed.

When an order between the plan's actions is needed, we can use yet another pattern, the "sequence" one (SEQ), defined by the operator $\Theta: A^{2} \rightarrow A$. We say that an action $A_{\text {res }}=A_{1} \Theta A_{2}$ is completed if $\forall j=1,2 \exists t_{j}>t_{0} \in$ Time and $t_{j}>t_{j-1}$ with the property that $A_{j}$ is completed starting with the $t_{j}$ and $A_{j+1}$ is activated at $t_{j}+1$ (Figure 14). Here $t_{0}$ represents the moment of the activation of the action $A_{l}$. In other words, the actions are activated and completed in the indicated sequence.

By constraining the action(s) completion into a time interval, we obtain the bounded versions of the above defined $A L L$, $F O F$, and $S E Q$ operators, as van Lamsweerde suggests (Lamsweerde \& Letier, 2000). We have found useful, in the action's plan expression, the use of n-ary extensions of these binary operators.

The action's activation corresponds with forcing $A_{\text {res }}$ started concept to $l$ in the action's plan. This means that the execution of the action's plan produces the acti- vation of component actions. In their turn, the corresponding effectors will be triggered.

If a component action fails, the whole plan fails in the case of $A L L$ and $S E Q$ patterns. By contrary, if the failed action participates in a FOF pattern, the plan is still active, and another action is waiting to succeed.

In the case of use of bounded version of above-mentioned operators, the action plan is ended if the plan is accomplished in the specified time interval, or stopped otherwise. More, at any moment, if the action's context became non-valid, the action is stopped.

Based on the action's plan, we are able to obtain different evaluations of its accomplishment level, either by counting its completed actions or by the means of the objective completed concept value. The greater this number, the greater may became the objective/action priority from the agent's perspective.

The action's corresponding FCM convergence to a fixed-point attractor assures the action's accomplishment or failure, depending on the value of completed concept at this point.

In our notation, we can express our tutor agent's global objective as $O=A L L$ ( $A C, F, A S C$ ), where we have noted by $A C$ the "avoid collision," by $F$ the "follow the child" and by ASC the "assist the child" objective. According to the proposed operators semantic, the tutor will evaluate and then activate the three objectives, based on their corresponding values in the FCM.

If there are some concurrent actions with similar effects, then they are let to cooperate; otherwise the action with lower priority is made inactive. In our case, the only concurrent actions may be $A C$ and $F$. For this, the value of the corresponding driving concept for $A C$, respectively for $F$, is 
given by the inverse of the distance to the object the tutor will try to avoid, respectively the child the tutor is following. After the currently active action will be accomplished, the rest of the actions will be reevaluated and activated accordingly.

We have to remind that the associated effectors control the values propagation through a fuzzy plan. This makes them different than but as simple as classical FCMs.

\section{IMPLEMENTATION}

Virtual environment and its components have been developed with Virtual Reality Modeling Language (VRML) ISO standard. Users may view 3D contents with a Web browser and a VRML plug-in. The actual implementation is using Cortona VRML plug-in from ParallelGraphics. The environment distribution is based on the DeepMatrix software (Reitmayr, Carroll, Reitmeyer \& Wagner, 1998) from GEOMETREK. This software enables users to enter $3 \mathrm{D}$ Web sites where they can interact with other users and objects. DeepMatrix implements client-server architecture.

On the server side, all messages are broadcasted in the same order to all clients. We have refined the proposed implementation from GEOMETREK, by introducing a filtering and pseudo-dead reckoning mechanism, which permit a more friendly and flexible connection of young users.

On the client side, interfaces have been implemented in Java language with a special attention due to the fact that users are viewing the HTML pages with Internet Explorer and running the Microsoft Virtual Machine. The use of External Authoring Interface (EAI) permits the client to achieve complex tasks by connecting the
VRML Web browser plug-in with a Java applet within the same Web page (Figure 15). The Java applet loads VRML content into the plug-in and adds avatar representation to the virtual world. The plug-in updates the Java applet about users' position and orientation in the virtual world.

There is one more type of information that is published between all the clients of a virtual world on DeepMatrix server, the other shared information, like pictures, sounds, and strings. Generally, everything is declared as shared node in VRML files. By doing this, we can reach an optimised solution for a distributed virtual environment, with non-shared information downloaded locally.

\section{VR Agents}

The virtual agents were designed on an approximate-body approach (Thalmann, 2000), which frequently provides position and orientation information to remote hosts, taking into account a minimal set of joint points. Their geometrical structure components were implemented in VRML using a PROTO structure. Behaviours were designed with the 3DStudioMax software, and then exported to VRML format. The resulting code was finally inserted into the agent's PROTO structure.

The environment dynamics is perceived by the agent itself through its Perception module (Figure 9). Based on VRML sensor-like components, this module uses VRML eventIn mechanism in order to transmit the detected stimuli to the agent's decisional module. And because the agent itself has VRML components in the VRML environment, using these sensors it is able to realise the exteroception as well as the interoception. Once the decision is made by the agent's Decisional module, which was implemented using Java 
Figure 15: Client-Server Architecture

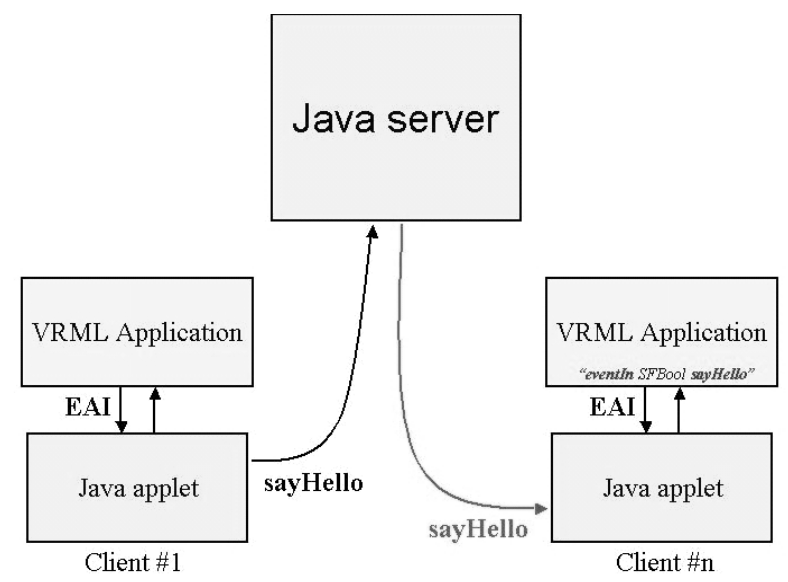

classes, it sends execution commands to its Action module. This time, we are using the VRML eventOut mechanism in order to activate the agent's behaviours (Figure $15)$.

The implemented behaviours are walk, turn left, turn right, standby, look at, laughing, drag/drop object, greetings such as "Hello!", "Good Bye!", "Help me!", "Thank you!", "I agree" and "I disagree". These VR agent's atomic actions form agent's capabilities and are used in the action plan realisation. The child may select a behaviour, which will be broadcasted (gesture and sound) to other children through its personal avatar.
In order to assure the EVE's agents adaptability to their dynamic environment we are using the following plans:

AgentGoal=ALL(Play,AvoidCollision,Help) Play $=S E Q\left(\right.$ Room Completed,Room $_{2}$ Completed) Room Completed=AL(Sentence, Sentence, Sentence) Sentence $_{i}=S E Q\left(A \amalg\left(\right.\right.$ Label $_{1}, \ldots$, Label $\left._{5}\right)$, Valid_Sentence $)$ Label $_{k}=$ action to place the Label $_{k}$ into a label socket from agent's walls.

Valid_Sentence=is an example of requested action (which depends on other agents' actions), in our case on tutor decision on proposed Sentence, which is part of tutor's Assist plan.

\section{Figure 16: Virtual Tutor in EVE Environment}

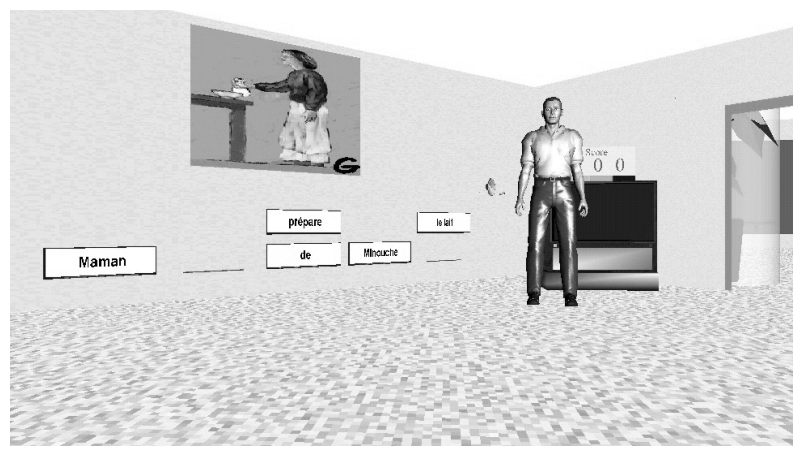


Room2Completed=SEQ(AL(Img,,.. $.1 \mathrm{mg})$ ),Valid_Story) $I m g_{i}=$ action to place the Image into an image socket from common room green wall.

Valid_Story=is another tutor action which participate as requested action in a fuzzy plan. AvoidCollision is considered as an atomic action. For its corresponding FCM and use of other atomic actions (Figure 11).

\section{Help=FOF(Ask_for,Give)}

Ask_for $=$ explicit demand of other agents to help the current one in its task.

Give $=$ SEQ(Approach, Label) means that the agent will try to approach to the agent who has sent the Ask_for demand. After that, it will try to place a Label into the right socket. This time, the action plan components are demanded actions for all the other agents, except the agent asking for help.

This is the most frequent situation in which the cooperation between agents appears. Once an agent sends the Ask_for help demand, all the agents in its environment will receive this demand under the form of an external stimulus and will activate their Give action plan. Due to the environment's dynamic (agents positions, access paths, obstacles, etc.), only one of these agents will succeed to accomplish this task. The other agents will be informed about this and they will skip the Give plan and will decide on the next action according to environment context. This way, we have eliminated the obsolete agent's actions due to environment changes during the time interval between agent's action decision and action completion.

More, using virtual agents we are able to ensure the persistence of the game. Let us suppose that there is a child who wants to quit the game before its end. In this case, without a virtual agent who takes the child's place, the game will be blocked. But using a virtual agent, the child can quit the game without affecting its flow, its role being switched to the corresponding virtual agent.

\section{Virtual Tutor}

Because of the number and distribution of children that are playing the game, the teacher is unable to assist them all the time. More, some children may need additional assistance from their teacher. For this, we have introduced the virtual tutor in EVE environment (Figure 16). The role of virtual tutor is not to substitute the real teacher but to encourage the child to test the environment even in the absence of its real teacher.

A virtual tutor is basically a virtual agent which has as objective to assist the child in its training. He or she can do this by recalling one sentence the child has to reconstruct or the whole story. He or she can do all this at user's demand or he/she can decide to act based on user actions (label's erroneous movements). More, he is able to move, avoid collisions, salute, confirm or infirm user's choices.

The tutor's plans are extension of those described in the previous subsection:

TutorGoal=ALL(Goal,AvoidCollision,Assist) Assist=SEQ(Approach,FOF(GiveAdvice,Valid_ Sentence,Valid_Story)), (Fig.11). GiveAdvice=FOF(Say_sentence, Say_story), depending on the assisted agent actions, the tutor will give some suggestions.

In the tutor's Goal plan, the actions like RoomCompleted, Sentence or Label are requested actions for the existing agents. The teacher may use the virtual tutor's avatar and so it will be able to realise remote assistance for the children using EVE. In this case, the virtual tutor's behaviour will be much more complex from a decisional point of view, because there will be 
a real person who interacts, but will still follow the actions plan.

\section{CONCLUSION \& FUTURE WORK}

In this paper we have presented a virtual environment specially dedicated to pedagogical purposes. This virtual environment is accessible with a standard Web browser and VRML plug-in without any specific or additional software. The application is successfully in use as a complement of traditional learning exercises in primary schools from France, Morocco, Romania and Japan. Such exercises already exist using scissors and paper. But the difference here is twofold:

- Children get an immediate validation of their works; for example an animal appears when the sentence is correct;

- Children do not realise that they are working; in fact they feel as if they were playing together.

Experiments have pointed out that games increase children's motivation, and new technologies such as virtual reality increase children's autonomy. More, they are able to take over the virtual world and to move into the virtual space. For example, after a short time of practice, children have transposed into the virtual world one of their real games: "hide-and-seek". The cooperative work demonstrates that children in a multicultural framework are able to work together if they comply with common rules. It is important to remember that a computer is only a machine, which is programmed to provide positive encouragements and congratulations so that children can consider it as a friend. On the one hand, it encourages children to be more active so that they dare answer something; it enables children to make the exercise as many times as necessary. On the other hand, it enables children to go faster and then increase the number of exercises.

The second game (Figure 3 ) is meant to help with text understanding and logic. Generally children have difficulties reading and to correcting themselves. They often make the same mistakes. Here, it is impossible because they have to change something if they are wrong; they have to build a new reasoning. This points out that our application contributes to increasing the ability of children to reason by themselves.

On a pedagogical point of view, planned for the primary courses, EVE is now adaptable for older children. The teacher may pass from a text comprehension to grammar or syntax studies. More, new applications should be developed in other domains of interest such as: foreign languages, geometry, algebra, and others. A special benefit of the authoring tools lies in the fact that the teacher can easily create new exercises. Especially, the teacher can design exercises that fit with children's interest. Consequently, it strengthens children's attention to exercises contents. As stories are shared between classrooms or schools, it increases the quantity of exercises teachers can propose to children.

On a technical point of view, current experiments will help us to design and implement new avatar behaviours according to end users' needs. We are also working on a more sophisticated mechanism, which is the integration of streaming audio and video into the virtual world. This should increase the performance of children's cooperative work. A new direction would be to use a platform independent interface, ARéVi. Doing so, we could reach a total independence together with a higher performance in 3D rendering. More, we are interested in emotional and social aspects 
of agents' behaviour. Validation of critical action plans, like cyclic ones, is one of our priorities. For short term, we intend to let the EVE's tutor assist more children in the same time, to participate into the voting mechanism, or even to make some mistakes in order to supplementary test the children, as a higher level of children's pedagogical evaluation.

To conclude, we believe that virtual reality is likely to enable the development of new products that will help children and teachers in their tasks of learning and teaching through friendly interfaces. Finally, cooperative experiments pointed out that such cooperative work introduces a kind of subliminal target, which is: "training children in learning democracy".

\section{ACKNOWLEDGMENTS}

Thanks are due to the Intergovernmental Agency of the Francophonie, which funded the EVE project in the framework of the Francophone Information Highway Fund, the Brittany Regional Council, which funded this work in the framework of the MEGALIS Project, and the HewlettPackard Company. Thanks are also due to primary schools Kroas Saliou, Plouzané, France, Dan Barbilian, Constanta, Romania, El Khalil, Casablanca, Morocco, and Ootsukadai elementary school, Yokosuka, Japan, and companies Virtualys, Brest, France, Impromex, Constanta, Romania, and TBE MAROC, Rabat, Morocco involved in the project.

\section{REFERENCES}

Bates, J. (1992). Virtual reality, art and entertainment. PRESENCE: Tele-operators and Virtual Environments, 1(1), 133-138.
Benford, S.D., \& Fahln, L.E. (1993). A spatial model of interaction in large virtual environments. Proc. ECSCW'93 (pp. 109-124). Milano, Italy: Kluwer Academic.

Bobick, A., Intille, S., Davis, J., Baird, F., Pinhanez, C., Campbell, L., Ivanov, Y., Schutte, A., \& Wilson, A. (2000). The KidsRoom - A perceptually-based interactive and immersive story environment. Presence: Teleoperators and Virtual Environments, 8(4), 367-391.

Bruner, J. (1986). Actual Minds, Possible Worlds. Cambridge, MA: Harvard University Press.

Cavazza, M., Charles, F., \& Mead, S.J. (2002). Interacting with virtual characters in interactive storytelling. ACM Joint Conference on Autonomous Agents and Multi-Agent Systems (pp. 318-325). Bologna, Italy.

Druin, A. (1999). Cooperative inquiry: Developing new technologies for children with children. Proceedings of Human Factors in Computer Systems (chi99) (pp. 223-230). ACM Press.

Druin, A., Revelle, G., Bederson, B., Hourcade, J., Farber, A., Lee, J., \& Campbell, D. (2003). A collaborative digital library for children: A descriptive study of children's collaborative behaviors and dialogue. Journal of ComputerAssisted Learning, 19(2), 239-248.

El-Nasr, M.S., Yen, J., \& Ioerger, T. (2000). Flame - A fuzzy logic adaptive model of emotions. Autonomous Agents and Multi-Agents Systems, 3, 219-257.

Hayes-Roth, B., \& vanGent, R. (1997). Story-making with improvisional puppets. Proceedings of First International Conference on Autonomous Agents (pp. 1-7).

Hefco, V. (1997). The Physiology of Animals and Humans (in Romanian). Bucharest, Romania: Ed.D.P. 
Herrero, P., \& Antonio, A.D. (2002). A human based perception model for cooperative intelligent virtual agents. (COOPIS'02) $10^{\text {th }}$ International Conference on Cooperative Information Systems (pp. 195-212). Irvine, CA, USA.

J@ ardin (2004). Jardin Botanique de Montréal. Online: http://www.ville. montreal.qc.ca/jardin/jeunes

Johnson, A., Moher, T., Cho, Y., Lin, Y., Haas, D., \& Kim, J. (2002, March/ April). Augmenting elementary school education with vr. IEEE Computer Graphics and Applications, 6-9.

Johnson, A., Moher, T., Leigh, J., \& Lin, Y. (2000). Quickworlds: Teacher driven VR worlds in an elementary school curriculum. Proceedings of SIGGRAPH 2000 - Educators Program, New Orleans, LA.

Johnson, A., Moher, T., Ohlssoon, S., \& Gillingham, M. (1999). The Round Earth Project - Collaborative VR for conceptual learning. IEEE Computer Graphics and Application, Virtual Reality, 19(6).

Kosko, B. (1997). Fuzzy Engineering. Prentice Hall.

Lamsweerde, A. van, \& Letier, E. (2000). Handling obstacles goal oriented requirements engineering. IEEE Trans. on Software Engineering, 26(10), 9781005.

Mantovani, F. (2001). VR learning: Potential and challenges for the use of $3 \mathrm{D}$ environments in education and training. Towards CyberPsychology: Mind, Cognitions and Society in the Internet Age.

Marshall, P., Rogers, Y., \& Scaife, M. (2002). Puppet: A virtual environment for children to act and direct interactive narratives. Second International Workshop on Narrative and Interac- tive Learning Environments, Edinburgh.

Mateas, M. (1997). An OZ-centric view of interactive drama and believable agents. (Tech. Rep.), CMU-CS-97-156.

Montemayor, J., Alborzi, H., Druin, A., Hendler, J., Pollack, D., Porteous, J., Sherman, L., Afework, A., Best, J., Hammer, J., Kruskal, A., Lal, A., Plaisant-Schwenn, T., Sumida, L., \& Wagner, R. (2000, April). From pets to storykit: Creating new technology with an intergenerational design team. Workshop on Interactive Robotics and Entertainment (WIRE-2000), Pittsburgh.

Pantelidis, V. (1995). Reasons to use virtual reality in education. VR in Schools, 1(1), 9 .

Parenthoen, M., Reignier, P., \& Tisseau, J. (2001). Put fuzzy cognitive maps to work in virtual worlds. Fuzz-IEEE01, Melbourne, Australia.

Piaget, J. (1929). The Child's Conception of the World. New York: HarcourtBrace.

Popovici, D.M., Serbanati, L.D., \& Gerval, J.P. (2003). Agent-based modeling of virtual environments. Proceedings of VRIC 2003 (pp. 149-158). Laval, France.

Popovici, D.M., Serbanati, L.D., \& Harrouet, F. (2003). The virtual environment - Another approach. WSCG'2003 Posters Proceedings (pp. 109-112). Plzen, Czech Republic.

Prada, R., Machado, I., \& Paiva, A. (2000). TEATRIX: Virtual environment for story creation. Intelligent Tutoring Systems (pp. 464-473).

Reitmayr, G., Carroll, S., Reitmeyer, A., \& Wagner, M. (1998, October 30). Deepmatrix - An Open Technology Based Virtual Environment System.

Robertson, J. (2002). Computer games For better or for worse? i3 Magazine, 
$12,32-34$.

Roussos, M., Johnson, A., Moher, T., Leigh, J., Vasilakis, C., \& Barnes, C. (1999). Learning and building together in an immersive virtual world. Presence, 8(3), 247-263.

Thalmann, D. (2000). Challenges for the research in virtual humans. Workshop Achieving Human-like Behavior in Interactive Animated Agent, Barcelona, Spain.

Tisseau, J., \& Harrouet, F. (2003). Autonomie des entités virtuelles. In P. Fuchs (Ed.), Le traité de la réalité virtuelle, 2, 85-124. Les Presses de l'Ecole des Mines, Paris.

Volbracht, S., Domik, G., BackeNeuwaldand, D., \& Rinkens, H. (1998). The "City Game" an example of a virtual environment for teaching spatial orientation. Journal of Universal Computer Science, 4(4), 461-465.

Winn, W. (1993). A conceptual basis for educational applications of virtual reality. Technical Report TR 93-9.

Youngblut, C. (1998). Educational uses of virtual reality technology. Technical Report IDA Document D-2128.

Dorin-Mircea Popovici is a PhD student in Computer Science at "Politehnica" University at Bucharest, and a lecturer at OVIDIUS University (Constanta, Romania). His PhD subject concerns the space modeling in virtual universes. He is interested in computer graphics, computational geometry, virtual space modeling, virtual physiology and multi-agent environments. Since 2003, he has been an associated researcher at CERV as manager of EVE project.

Jean-Pierre Gerval obtained his Doctorate in Automation from the University of Valenciennes in France (1987). He has been project manager at the "Institut d'Informatique Industrielle" in Brest (1988-2003) and associate professor of Computer Sciences at the "Ecole Nationale d'Ingénieurs de Brest" (1993-2003). He is currently head of the Computer Sciences Department at the "Institut Supérieur de l'Electronique et du Numérique" in Brest. His research interests include distributed virtual reality and virtual environments especially dedicated to pedagogical applications.

Pierre Chevaillier is a professor assistant in Computer Science and works at the CERV. He works on the use of the multiagent paradigm to design digital simulations where users have to play an active and collaborative role. He is the leader of the MASCARET project and focuses its research activities on the interaction and organisational facets of heterogeneous multiagents systems (where both artificial and human agents are involved).

Jacques Tisseau is a professor in Computer Science and is head of LI2/ENIB and CERV. His domains of interest are entity's autonomy in virtual environments, and "in virtuo" experimentation in the modeling processes.

Luca-Dan Serbanati is a professor of Computer Science at the Ovidius University of Constanta and an IT adviser, teacher, and mentor for industry in Italy. He is the author of the book Integrating Tools for Software Development, where multi-facetted models for the software process are introduced. Serbanati received a PhD in Computer Science from the Politehnica University of Bucharest. His research interests address systems model-driven analysis and design, formal models, distributed systems, and software development paradigms. 
40 Journal of Distance Education Technologies, 2(4), 18-40, Oct-Dec 2004

Patrick Gueguen is a primary school teacher. He is the headmaster of Plouzané Primary School, France. One of his main targets is to open his school and encourage children to use new technologies. He takes part in the pedagogical aspects of EVE project, being involved in it from its beginning. 